\title{
Role of Plexin B1 in a Breast Cancer Cohort of Pakistani Patients and its Contribution Towards Cancer Metastasis as Indicated by an In Vitro Model
}

\author{
MUHAMMAD FARAZ ARSHAD MALIK ${ }^{1,2}$, SYEDA KIRAN RIAZ $^{1}$, S.H. WAQAR ${ }^{3}$, \\ FARHAN HAQ ${ }^{2}$, LIN YE ${ }^{1}$ and WEN G. JIANG ${ }^{1}$ \\ ${ }^{1}$ Cardiff University-Peking University Cancer Institute (CUPUCI), \\ Cardiff University School of Medicine, Cardiff University, Cardiff, U.K.; \\ ${ }^{2}$ Department of Biosciences, COMSATS-Institute of Information Technology, Islamabad, Pakistan; \\ ${ }^{3}$ Shaheed Zulfiqar Ali Bhutto Medical University/Pakistan Institute of Medical Sciences, Islamabad, Pakistan
}

\begin{abstract}
Background/Aim: In the current study, the role of plexin B1 in breast cancer metastasis was explored. Materials and Methods: Freshly-excised tumours along with background tissues of affected patients (n=121) were collected from Pakistani hospitals and processed for RNA isolation and $c D N A$ synthesis. Using quantitative polymerase chain reaction, expression of plexin $B 1$ was evaluated and correlated with clinicopathological parameters. Furthermore, involvement of plexin B1 in metastasis was explored by generating gene knockdown in MDA-MB-231 and MCF-7 breast cancer cells. Results: Poorlydifferentiated tumours showed low plexin B1 expression in comparison to well-differentiated ones. Similarly, reduced plexin B1 expression correlated positively with advanced tumour stage and metastasis. Loss of plexin B1 significantly reduced cell adhesion in comparison with respective control cell lines $(p<0.05)$. Knockdown of plexin B1 in MDA-MB231 cells led to a remarkable increase in cell motility in contrast to the respective control. Conclusion: Loss of plexin B1 expression might play a pivotal role in enhancing the metastatic potential of breast cancer cells.
\end{abstract}

Breast cancer is ranked among the top three most commonly prevailing types of cancers among women. According to Cancer Research UK, incidence rates for breast cancer are projected to increase by $2 \%$, with an expected increase of 210 new cases per 100,000 females from 2014-2035 (1).

Correspondence to: Professor Wen G. Jiang, Cardiff UniversityPeking University Cancer Institute (CUPUCI), Cardiff University, Cardiff, Wales, U.K. E-mail: JiangW@cardiff.ac.uk

Key Words: Plexin B1, breast cancer, qRT-PCR, adhesion, migration, metastasis.
According to the Karachi Cancer Registry data (2010-2015), breast cancer was the most frequently recorded malignancy (age-standardized incidence rate: 87.9) with highest incidence in Pakistan compared to all other Asian populations (2). Tumor metastasis is considered a leading cause of cancer-related death.

Numerous genetic, epigenetic and environmental/lifestyle factors are involved in disease progression. Interestingly, molecules responsible for early organ development and neuronal growth during embryogenesis may also contribute towards tumourigenesis. Invasion and metastatic complications are causes of increased morbidity. The role of semaphorins and plexins in tumor progression acting as axonal guidance cues has also been established (3). Regarding the similarity of molecular cross talk existing in both neural and non-neural cells, the role of these molecules in determining the ultimate fate of cancer has been extensively explored (4).

Based on sequence similarities, nine plexin proteins are grouped into four families, plexins A to D. The plexin-B family contains three members, plexin B1, B2 and B3 (5). Plexin B1 is a single transmembrane protein acting as a binding receptor for semaphorin 4D (6). It comprises of 2,135 amino acids encoded from 38 exons and is responsible for mediating semaphorin 4D signals via activated Ras-related C3 botulinum toxin substrate (RAC) (7). Plexin B1 is observed in both neuronal and non-neural tissues, including mesenchymal cells of kidney, tooth enamel, and epithelial cells of the olfactory system, oral cavity, lung and intestine (8).

Previously, loss of plexin B1 in patients with breast cancer was found to be directly correlated with poor overall survival, and local recurrence (9). Similarly, in ovarian, renal and skin cancer, altered plexin B1 expression has been correlated with advanced tumour grade, lymph node involvement and poor prognosis (10-12). In the present study, expression of plexin B1 in breast cancer cohort of a 
Pakistani population and its putative involvement in metastasis was explored. Furthermore, the effect of plexin B1 on cell adhesion and motility was also investigated using breast cancer cell lines.

\section{Materials and Methods}

Identification and collection of breast cancer biopsies. Approval from the Biosafety and Ethical Committees of Institute and concerned hospitals (PIMS at Islamabad, Holy Family Hospital at Rawalpindi) were obtained for the Pakistani cohort (Project approval no. 2989). Patients with breast cancer $(n=121)$, were included in this study, with age ranging from 23 to 75 years. Tumour along with neighbouring background tissues were excised by surgeons and immersed in RNAlater ${ }^{\circledR}$ (Thermo Scientific, Waltham MA, USA) solution. These samples were transferred to laboratory for immediate RNA extraction or storage at $-20^{\circ} \mathrm{C}$.Information related to age at disease onset, area affected, menopausal status along with pathological findings such as tumour grade, stage and patient's prognosis were also collected.

Extraction of RNA and synthesis of cDNA. RNA was isolated from both tumor and control tissues using TRIzol ${ }^{\circledR}$ (Invitrogen, Carlsbad, CA, USA) as mentioned earlier (9). After extraction, RNA was dissolved in diethyl pyrocarbonate (DEPC)-treated autoclaved water and stored at $-80^{\circ} \mathrm{C}$ until further use. RevertAid First Strand cDNA Synthesis Kit (Thermo Scientific) was used to synthesize cDNA strand from isolated RNA as per manufacturer's instructions.

Analysis of gene expression in breast cancer cohort. Plexin B1 (forward: 5'-GAGGTGGCCTACATCGAG-3', reverse: 5'-AGTGGT CTGAGCCACAGG-3') Ki67 (forward: 5'-GCCTTGGTCTCTTG GGAATAC-3', reverse: 5'-GGAGATTAGGAGCCAGTTTGAG-3') and $\beta$-actin (forward: 5'-ATGATATCGCCGCGCTCA-3', reverse: 5'CGCTCGGTGAGGATCTTCA-3') (internal housekeeping gene) transcript-specific primer sequences were designed using the Primer 3' software (13). Primers for the aforementioned genes were synthesized from Macrogen (Seoul, Korea). Reaction conditions for polymerase chain reaction (PCR) were: initial denaturation at $95^{\circ} \mathrm{C}$ for $15 \mathrm{~min}$, followed by 40 cycles at $95^{\circ} \mathrm{C}$ for $15 \mathrm{~s}, 53^{\circ} \mathrm{C}$ for $1 \mathrm{~min}$ and final hold at $4^{\circ} \mathrm{C}$. For accuracy, a triplicate dataset regarding mean copy number of genes was generated for the given cohort. Relative quantification of these molecules was obtained by using Livak's $2^{-(\Delta \Delta C T)}$ method (14).

Cell culture conditions. MCF-7 and MDA-MB-231 breast cancer cell lines were purchased from the American Type Culture Collection (Manassas VA, USA). These cell lines were maintained in normal Dulbecco's modified Eagle's medium supplemented with $10 \%$ foetal bovine serum, penicillin and streptomycin. These cell lines were maintained at $37^{\circ} \mathrm{C}$, in $5 \% \mathrm{CO}_{2}$ and $95 \%$ humidity until further usage.

Knockdown of plexin B1 expression in breast cancer cell lines. Ribozyme-based knockdown of plexin B1 was achieved using a previously published procedure (15). Briefly two sets of small hammerhead ribozymes were synthesized targeting plexin B1 and transfected separately into MCF-7 and MDA-MB-231 cells using a pcDNA3.1/nt-GFP-TOPO ${ }^{\circledR}$ vector (Invitrogen, Paisley, UK). After orientation check, these clones were transformed via electroporation and empty vectors (lacking ribozymes) were forcefully introduced as control. MDA-MB-231 cells containing only vector were termed as MDA-MB-231CT, while those containing ribozymes were termed as MDA-MB-231PBR1 and MDA-MB-231PBR2, respectively. Similarly, MCF-7 cells containing only empty vector were labelled as MCF-7CT while those containing ribozymes were termed as MCF-7PBR1 and MCF-7PBR2.

Confirmation of plexin B1 knockdown in cancer cell lines. Expression profiling of plexin B1 in all aforementioned cell lines was evaluated using qRT-PCR. Upon reaching above $90 \%$ confluence, total RNA isolation from the cell lines was carried out using TRI reagent (Sigma-Aldrich, Darmstadt, Germany). Briefly, cells were collected in $1.5 \mathrm{ml}$ microfuge tubes and suspended in TRI reagent. After sequential treatment with chloroform and isopropanol, total RNA was precipitated. RNA retrieved from each cell line was quantified after being dissolved in DEPC-treated water. Synthesis of cDNA was carried out using an RT kit with an anchored oligo (dT) primer supplied by AbGene (Thermo Scientific, Rockford, IL, USA). Glyceraldehyde 3-phosphate dehydrogenase (GAPDH) was used as an internal control using forward primer 5'-CAGGAGGTT GAAGGACTAAA-3' and reverse primer 5'-GGGATCAGTTTTC TTTGTCA-3'. Reaction conditions were initial denaturation at $95^{\circ} \mathrm{C}$ for $5 \mathrm{~min}$, followed by 30 cycles of $95^{\circ} \mathrm{C}$ for $30 \mathrm{~s}, 55^{\circ} \mathrm{C}$ for $30 \mathrm{~s}$, $72^{\circ} \mathrm{C}$ for $1 \mathrm{~min}$, and a final extension step at $72^{\circ} \mathrm{C}$ for $10 \mathrm{~min}$. Amplified products were assessed using $2 \%$ agarose gel electrophoresis for plexin B1. MDA-MB-231PBR2 and MCF7PBR2 cells were selected for subsequent experiments based on their remarkable down-regulation of plexin B1.

Effect of plexin Blon cell adhesion and motility. Effects of plexin B1 on cancer cell adhesion and motility were evaluated using already established protocols $(15,16)$.

Cancer cell adhesion assay: Briefly, a 96-well flat-bottom plate was used for this assay. Matrigel ${ }^{\mathrm{TM}}(5 \mu \mathrm{g})$ (BD Biosciences, San Jose CA, USA) dissolved in $100 \mu \mathrm{l}$ of serum free medium was dispensed into each well of the plate. After drying and rehydration, these pre-coated wells were used for cell attachment. A total of 45,000 cells from each cell line were seeded separately into each well of the Matrigel-coated plate. After incubation of $40 \mathrm{~min}$, cells were aspirated out without disrupting adherent cells. The number of cells remaining adhered to basement membrane were counted under light microscope after standardized crystal violet staining.

Cell motility assay: Briefly, cells from each cell line were seeded in a 96-well flat-bottom plate for monolayer formation. After incubation for $24 \mathrm{~h}$ at $37^{\circ} \mathrm{C}$, wounding in the cell layer was introduced to measure the wound-healing migration distance. Photographs taken at $0,30,60,90,120$ min after wounding were analysed using Image $\mathrm{J}$ software (University of Wisconsin-Madison, Madison WI, USA) (17).

Data analysis. Wilcoxon signed-rank test was applied to test significance between paired tumors and adjacent normal controls. Mann-Whitney $U$-test and Kruskal-Wallis ANOVA were applied to test for the association of gene expression with clinicopathological parameters. All functional assays were performed in triplicate sets with a minimal repetition of at least three. Empty vector-containing breast cancer cell lines MDA-MB-231CT and MCF-7CT were taken as controls and compared with MDA-MB-231PBR2 and MCF- 
7PBR2 cell lines, respectively. Two tailed $t$-test was used for detecting any probable effect of plexin $\mathrm{B} 1$ on cell motility and adhesion. GraphPad Prism 5 (GraphPad Software, Inc., La Jolla CA, USA) was used to assess significance of differences between cell lines and differences were considered significant at $p<0.05$.

\section{Results}

Demographic features of breast cancer cohort. Patients with breast cancer in this cohort from Pakistan were more commonly $\leq 45$ years of age $(n=63)$ rather than $>45$ years old $(n=58)$. Similarly, patients were more frequently premenopausal than postmenopausal. Interestingly in this cohort, the highest proportion of tumours were moderately differentiated $(n=75)$ followed by poorly differentiated and well-differentiated tumour specimens ( $n=34$ and $n=11$, respectively), indicating the prevalence for delayed consultancy in this population.

Plexin B1 expression. Plexin B1 expression was lower by more than twofold in tumours in comparison to their respective normal tissue ( $p=0.0006$; Table I). Based on tumour grading, plexin B1 expression was significantly down-regulated among patients with poorly differentiated tumours (grade III; $0.5 \pm 0.8, p<0.05$ ) as compared to those with well-differentiated ones. Plexin B1 expression was highly significantly $(p<0.05)$ negatively correlated with tumour stage in this Pakistani breast cancer cohort. Similarly, reduced expression of plexin B1 also correlated significantly with increased metastatic potential (Figure 1; Table I). Strong positive correlation was observed between expression of plexin B1 and estrogen receptor/progesterone receptor $(E R / P R)$ in the cohort while there was no relationship with human epidermal growth factor receptor 2 (HER2) and proliferation marker Ki-67 (Table II). Expression of plexin B1 was also significantly reduced in the aggressive tumour subtypes such as HER2 $(p<0.0001)$ and triple-negative $(p<0.0001)$ as compared to luminal subtypes in patients with breast cancer (Figure 1; Table I).

Knockdown of plexin B1 expression in breast cancer cells. In order to assess the effect of plexin B1 on cellular functions, constructed plasmid vectors carrying anti-plexin B1 ribozymes were transfected into the two breast cancer cell lines. Following selection with blasticidin, RNA was extracted and the plexin B1 transcripts in the transfected cells were determined using RT-PCR. The knockdown of plexin $\mathrm{B} 1$ was verified in the respective transfectants and MDA-MB-231PBR2 and MCF-7PBR2 cells were chosen for further functional studies (Figure 2).

Effect of plexin B1 on cell adhesion. A direct correlation of plexin B1 expression on cancer cells attachment to basement membrane was established. Interestingly, significantly fewer
Table I. Correlation of plexin B1 expression with clinicopathological characteristics and molecular subtypes of breast cancer.

\begin{tabular}{|c|c|c|c|c|}
\hline \multirow[t]{2}{*}{ Variable } & \multirow[b]{2}{*}{ Subgroup } & \multirow[t]{2}{*}{ Total, $\mathrm{n}$} & \multicolumn{2}{|c|}{ Plexin B1 } \\
\hline & & & Mean \pm S.D. & $p$-Value \\
\hline \multirow[t]{2}{*}{ Tissue } & Tumour & 120 & $0.4 \pm 0.8$ & $0.0006^{*}$ \\
\hline & Control & 120 & $1.1 \pm 1.8$ & \\
\hline \multirow[t]{3}{*}{ Grade } & $\mathrm{I}$ & 11 & $2.1 \pm 2.0$ & $0.004^{\#}$ \\
\hline & II & 75 & $0.6 \pm 0.9$ & \\
\hline & III & 34 & $0.5 \pm 0.8$ & \\
\hline \multirow[t]{4}{*}{ Stage } & I & 28 & $1.1 \pm 1.9$ & $0.02^{\#}$ \\
\hline & II & 54 & $0.6 \pm 0.8$ & \\
\hline & III & 34 & $0.4 \pm 0.7$ & \\
\hline & IV & 4 & $0.01 \pm 0.03$ & \\
\hline \multirow[t]{3}{*}{ Nodal involvement } & None & 37 & $1.5 \pm 2.2$ & $0.46 \#$ \\
\hline & $\leq 4$ Nodes & 66 & $0.6 \pm 0.9$ & \\
\hline & $>4$ Nodes & 17 & $0.2 \pm 0.3$ & \\
\hline \multirow[t]{2}{*}{ Metastasis } & None & 116 & $2.5 \pm 3.4$ & $0.03^{\dagger}$ \\
\hline & Distant & 4 & $0.01 \pm 0.03$ & \\
\hline \multirow[t]{2}{*}{ Age at disease onset } & $\leq 45$ Years & 63 & $0.5 \pm 0.8$ & $0.1^{\dagger}$ \\
\hline & $>45$ Years & 57 & $0.6 \pm 0.8$ & \\
\hline \multirow[t]{2}{*}{ Menopausal status } & Pre & 64 & $0.4 \pm 0.8$ & $0.02^{\dagger}$ \\
\hline & Post & 56 & $0.6 \pm 0.8$ & \\
\hline \multirow[t]{4}{*}{ Subtype } & Luminal A & 13 & $3.0 \pm 3.1$ & $<0.0001^{\#}$ \\
\hline & Luminal B & 68 & $3.6 \pm 4.4$ & \\
\hline & HER2 & 18 & $0.09 \pm 0.2$ & \\
\hline & Triple-negative & 21 & $0.04 \pm 0.07$ & \\
\hline
\end{tabular}

Data are the mean \pm S.D. relative expression of tumors compared with their respective normal tissue controls. HER2: Human epidermal growth factor receptor 2. *Wilcoxon signed-rank test; ${ }^{\dagger}$ Mann-Whitney $U$-test; \#Kruskal-Wallis ANOVA.

MDA-MB-231PBR2 cells remained attached to the Matrigel in comparison to MDA-MB-231CT cells $(p<0.05)$. Similarly, MCF-7PBR2 cells were also less adherent in comparison to MCF-7CT cells $(p<0.05)$. Hence plexin B1 plays an important role in heterotypic cell interactions in both breast cancer cell lines (Figure 3A).

Effect of plexin B1 on cancer motility. Cancer cell motility was also affected by reduced plexin B1 expression. The distance migrated over $120 \mathrm{~min}$ by MDA-MB-231PBR2 cells $(p<0.05)$ was significantly higher than MDA-MB231CT cells. On the other hand, reduced expression of plexin $\mathrm{B} 1$ did not significantly reduce the migratory capacity of MCF-7PBR2 cells over $120 \mathrm{~min}$ compared to the MCF-7CT cells $(p>0.05)$ (Figure 3B and C).

\section{Discussion}

Metastasis is a non-random process involving loss of cancer cell adhesion from the primary tumour site, invasion across the extracellular matrix, entry into the blood or lymphatic 


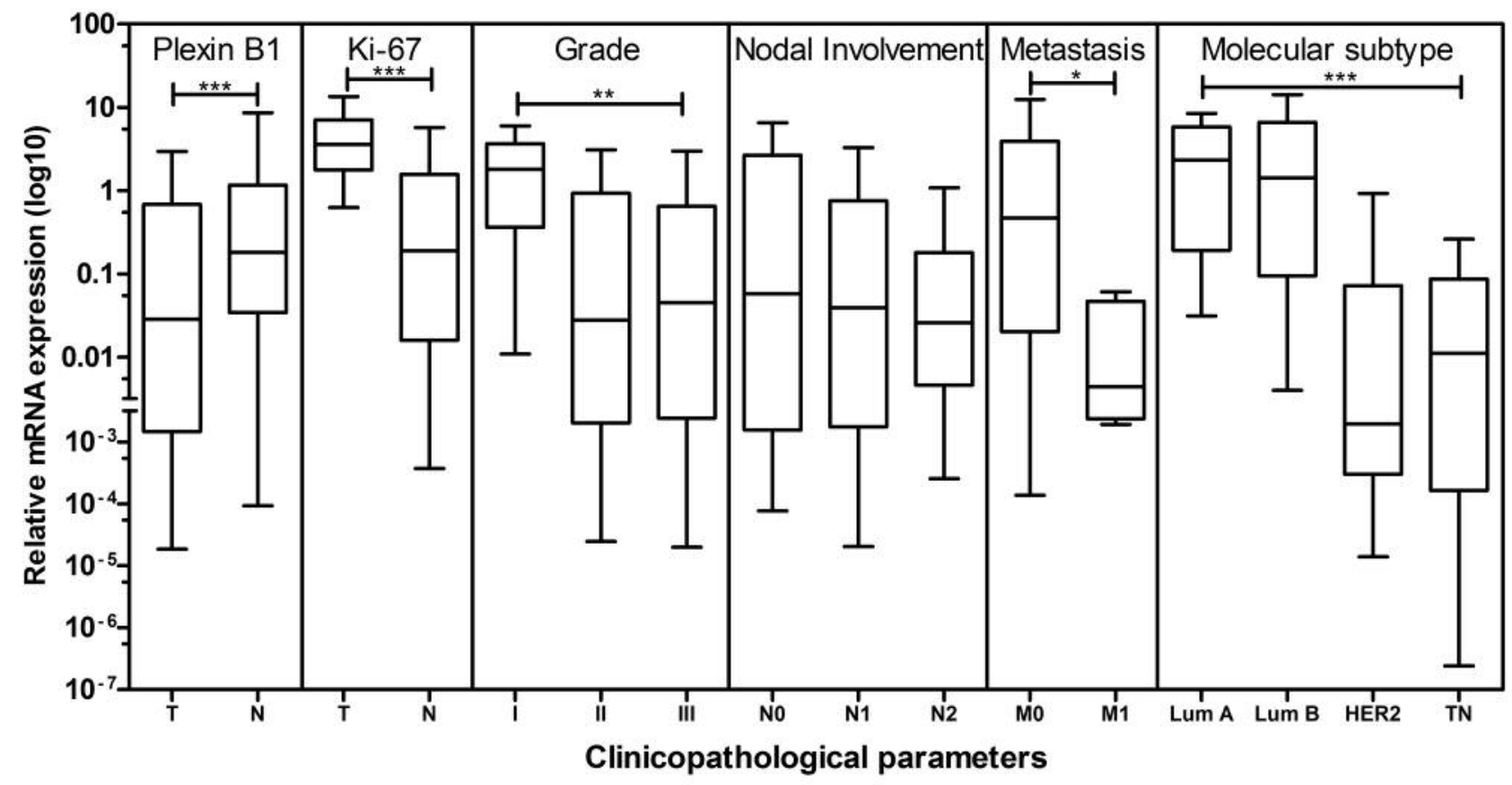

Figure 1. Correlation of expression of plexin B1 with clinicopathological parameters and molecular subtypes of the cohort. Expression was assessed using quantitative polymerase chain reaction and mRNA expression for all parameters was evaluated relative to adjacent normal controls using Livak's method. Significantly different at $* p<0.05$, $* * p<0.001$, and $* * * p<0.0001)$. T: Tumor; $N$ : normal tissue; N0:no nodal involvement; N1and N2: nodes involved; MO: no metastasis; M1: distant metastasis; Lum:luminal; HER2: human epidermal growth factor receptor 2; TN: triple-negative.

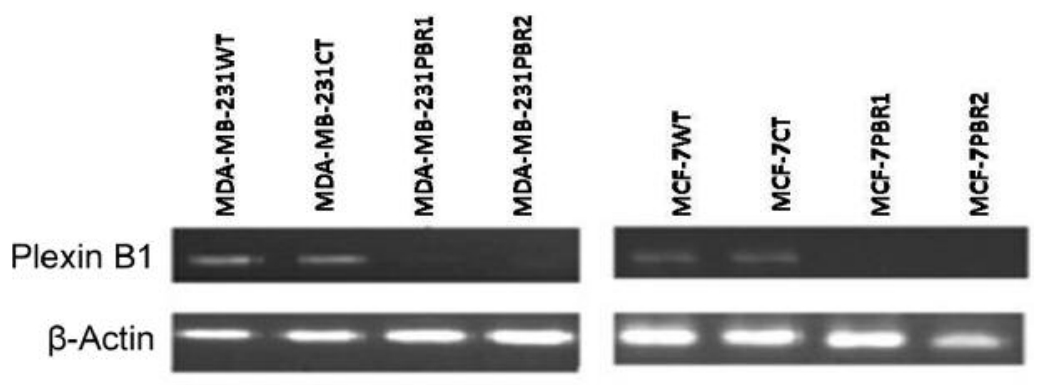

Figure 2. Confirmation of plexin B1 knock down in cancer cell lines using 2\% agarose gel; B-actin was used as control. WT: Wild type; CT: control; PBR1/PBR2: plexin B1 ribozyme 1/2.

channels, motility, compromising host immune response and extravasation at a suitable secondary site. The systematic spread and localization of cancer cells at secondary sites is mediated via several molecules. In breast, ovarian, renal and skin cancers, reduced plexin B1 expression was correlated with poor tumour grade, lymph node involvement and poor prognosis (10-12). Numerous receptors interact with plexin B1 for its downstream effector response inside the cell. Several studies suggest that reduced expression of plexin B1 was found to be significantly correlated with poor disease outcome, nodal involvement and metastasis in patients with breast and skin cancer $(11,18)$. On the contrary, plexin B1 interaction with various downstream effectors especially HER2 may provoke a pro-tumourigenic response, as observed in prostate (19) and cervical cancer (20). Furthermore, in ovarian cancer cases, co expression of MET proto-oncogene, receptor tyrosine kinase (MET) and plexin B1 was directly associated with metastasis and lymph node involvement (21). These contrasting findings are influenced by variability in plexin B1-mediated responses depending upon factors such as tissue specificity, nature of transducers and interacting molecules (22). 

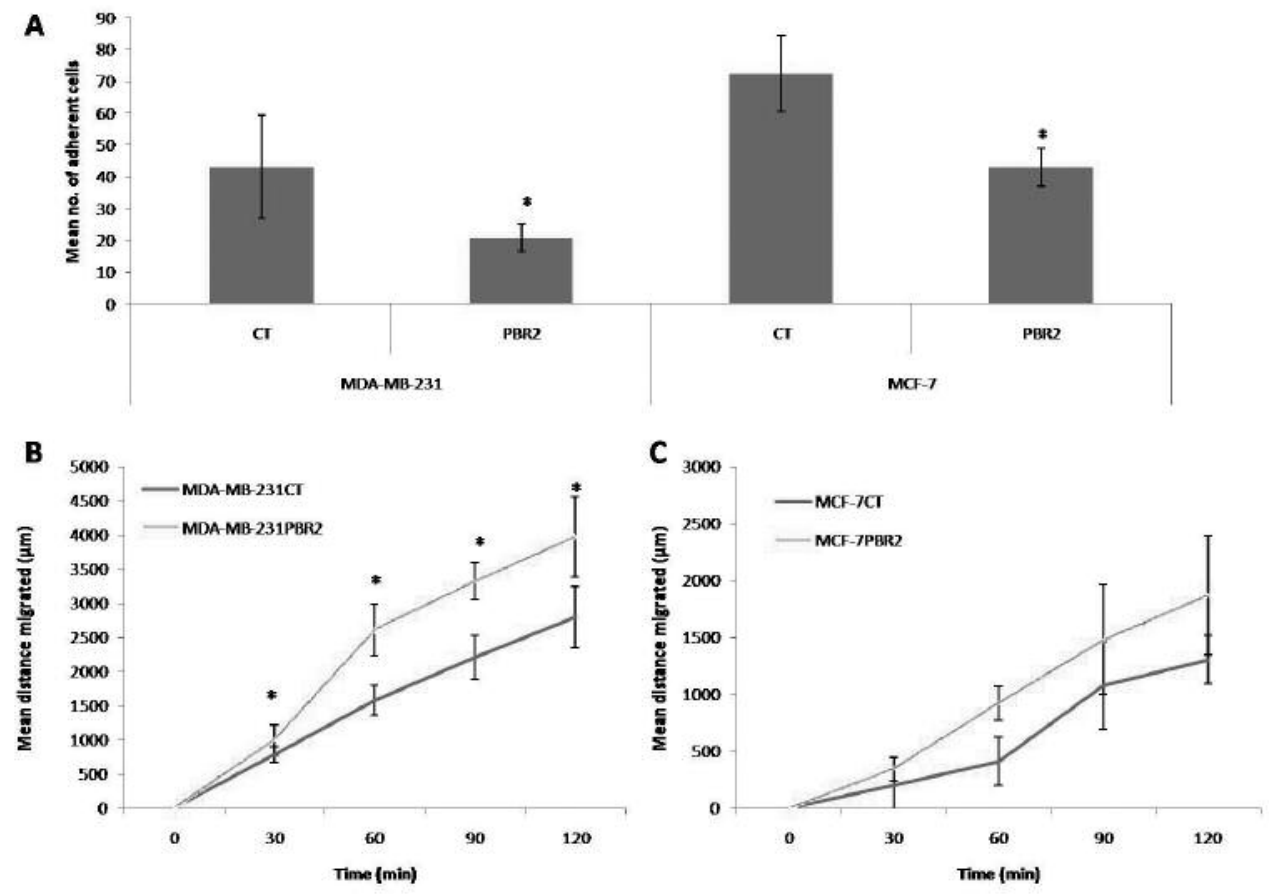

Figure 3. Effect of plexin B1 on adhesion (A) and motility of MDA-MB-231 (B) and MCF-7 (C) breast cancer cells. *Significantly different at p<0.05. CT: Control; PBR2: plexin B1 ribozyme 2.

Table II. Correlation (Spearman's R) of plexin B1 expression with estrogen receptor $(E R)$, progesterone receptor $(P R)$, human epidermal growth receptor (HER2) and proliferation marker (Ki67) positivity in the studied breast cancer cohort.

\begin{tabular}{lccrc}
\hline & ER & PR & HER2 & Ki67 \\
\hline Plexin & $\mathbf{0 . 5 3}$ & $\mathbf{0 . 4 8}$ & -0.03 & 0.13 \\
ER & & $\mathbf{0 . 9 1}$ & 0.11 & $\mathbf{0 . 2 6}$ \\
PR & & & 0.11 & $\mathbf{0 . 2 3}$ \\
HER2 & & & & 0.11 \\
\hline
\end{tabular}

Bold values represent correlation significant at $p<0.05$.

Knockdown of plexin B1 in both breast cancer cell lines here led to reduced cell adherence to Matrigel. It has been observed that plexin B1 triggers guanosine triphosphatase (GTPase) activity of Related RAS viral oncogene homolog (R-Ras) responsible for promoting cell adhesion and neural growth (23). Plexin B1 also triggers Ras homolog family member a (RhoA) activation upon exposure to semaphorin 4D while loss of semaphorin 4D led to Rho inactivation, leading to altered cytoskeleton (24). Thus, plexin B1 reduction significantly regulates tumour heterotypic interactions with stromal matrix. Binding of plexin B members with hepatocyte growth factor receptor (c-MET) and Erb-B2 receptor tyrosine kinase 2 (ERBB-2) triggers tyrosine phosphorylation, that leads to cell invasiveness and proliferation as has been observed in cervical and endothelial cell lines $(20,25)$. However, a contradictory finding pertaining to plexin B1 role in re-stricting tumour cell invasiveness was also observed in ovarian cancer (12).

Interestingly, loss of plexin B1 led to increased cell motility as observed in both breast cancer cell lines here. Mutations encompassing the extracellular portion of plexin B1 were found to regulate cell motility of prostate cancer cells (26). Reduced expression of plexin B1 was also observed to increase migration in melanoma cells via the vRaf murine sarcoma viral oncogene homolog B (B-Raf)/ putative mitogen activated protein kinase (MKK)/ extracellular signal-regulated kinase (ERK) axis (27). Interestingly plexin $\mathrm{B} 1$ has been shown to have pro-migratory effect on breast cancer cells by binding to ERBB2, and inhibits migration by binding to MET (28). These distinctive variations highlight a multifaceted role of plexin B1 varying among different tissues. Depending upon its localization, ligand binding variation and downstream effector molecular interaction play an ultimate decisive role in cancer metastasis.

Based on current findings, plexin B1 is suggested as a putative therapeutic target for restricting cancer spread. Further studies targeting downstream effectors of the plexin B1-mediated pathway can provide useful insights into the mechanistic details involved in breast cancer metastasis. 


\section{Acknowledgements}

The Authors are highly thankful to all patients, medical and technical staff support engaged in this study from Pakistan and UK. The Authors also would like to extend their gratitude to Higher Education Commission of Pakistan and Cancer Research Wales UK, for their valued approval and funding.

\section{References}

1 Breast cancer statistics. Available from: http://www.cancer researchuk.org/health-professional/cancer-statistics/statistics-bycancer-type/breast-cancer\#collapseZero [last accessed June 14, 2017].

2 Qureshi MA, Mirza T, Khan S, Sikandar B, Zahid M, Aftab M, Mohsin S, Sharafat S, Avesi L and Hassan S: Cancer patterns in Karachi (all districts), Pakistan: First results (2010-2015) from a Pathology based cancer registry of the largest government-run diagnostic and reference center of Karachi. Cancer Epidemiol 44: 114-122, 2016.

3 Malik MFA, Ye L and Jiang WG: The Plexin-B family and its role in cancer progression. Histol Histopathol 29: 151-165, 2014.

4 Chédotal A, Kerjan G and Moreau-Fauvarque C: The brain within the tumor: new roles for axon guidance molecules in cancers. Cell Death Differ 12: 1044-1056, 2005.

5 Maestrini E, Tamagnone L, Longati P, Cremona O, Gulisano M, Bione S, Tamanini F, Neel BG, Toniolo D and Comoglio PM: A family of transmembrane proteins with homology to the METhepatocyte growth factor receptor. Proc Natl Acad Sci USA 93: 674-678, 1996.

6 Kumanogoh A and Kikutani H: Semaphorins and their receptors: novel features of neural guidance molecules. Proc Jpn Acad Ser B Phys Biol Sci 86: 611-620, 2010.

7 Vikis HG, Li W and Guan K-L: The Plexin-B1/Rac interaction inhibits PAK activation and enhances Sema4D ligand binding. Genes Dev 16: 836-845, 2002.

8 Perälä NM, Immonen $\mathrm{T}$ and Sariola $\mathrm{H}$ : The expression of plexins during mouse embryogenesis. Gene Expr Patterns 5: 355-362, 2005.

9 Malik M, Ye L and Jiang W: Reduced expression of semaphorin 4D and plexin-B in breast cancer is associated with poorer prognosis and the potential linkage with oestrogen receptor. Oncol Rep 34: 1049-1057, 2015.

10 Gómez Román JJ, Garay GO, Saenz P, Escuredo K, Sanz Ibayondo C, Gutkind S, Junquera C, Simón L, Martínez A, Fernández Luna JL and Val-Bernal JF: Plexin B1 is downregulated in renal cell carcinomas and modulates cell growth. Transl Res 151: 134-140, 2008.

11 Stevens L, McClelland L, Fricke A, Williamson M, Kuo I and Scott G: Plexin B1 suppresses c-Met in melanoma: a role for plexin $\mathrm{B} 1$ as a tumor-suppressor protein through regulation of cMet. J Invest Dermatol 130: 1636-1645, 2010.

12 Ye S, Hao X, Zhou T, Wu M, Wei J, Wang Y, Zhou L, Jiang X, Ji L, Chen Y, You L, Zhang Y, Xu G, Zhou J, Ma D and Wang $\mathrm{S}$ : Plexin-B1 silencing inhibits ovarian cancer cell migration and invasion. BMC Cancer 10: 611, 2010.

13 Untergasser A, Cutcutache I, Koressaar T, Ye J, Faircloth BC, Remm $M$ and Rozen SG: Primer3 - new capabilities and interfaces. Nucleic Acids Res 40: e115-e115, 2012.
14 Livak KJ and Schmittgen TD: Analysis of relative gene expression data using real-time quantitative PCR and the $2^{-\Delta \Delta C T}$ method. Methods 25: 402-408, 2001.

15 Malik FA, Sanders AJ, Kayani MA and Jiang WG: Effect of expressional alteration of KAI1 on breast cancer cell growth, adhesion, migration and invasion. Cancer Genomics Proteomics 6: 205-213, 2009.

16 Jiang A, Zhao H, Cai J and Jiang WG: Possible effect of musclerelaxant anaesthetics on invasion, adhesion and migration of breast cancer cells. Anticancer Res 36: 1259-1265, 2016.

17 Schindelin J, Rueden CT, Hiner MC and Eliceiri KW: The ImageJ ecosystem: An open platform for biomedical image analysis. Mol Reprod Dev 82: 518-529, 2015.

18 Rody A, Holtrich U, Gaetje R, Gehrmann M, Engels K, Von Minckwitz G, Loibl S, Diallo-Danebrock R, Ruckhäberle E, Metzler D, Ahr A, Solbach C, Karn T and Kaufmann M: Poor Outcome in estrogen receptor -positive breast cancers predicted by loss of plexin B1. Clin Cancer Res 13(4): 1115-1122, 2007.

19 Damola A, Legendre A, Ball S, Masters JR and Williamson M: Function of mutant and wild-type plexin B1 in prostate cancer cells. Prostate 73: 1326-1335, 2013.

20 Qiang R, Wang F, Shi L-Y, Liu M, Chen S, Wan H-Y, Li Y-X, Li $\mathrm{X}$, Gao S-Y, Sun B-C and Tang H: Plexin-B1 is a target of miR214 in cervical cancer and promotes the growth and invasion of HeLa cells. Int J Biochem Cell Biol 43: 632-641, 2011.

21 Valente G, Nicotra G, Arrondini M, Castino R, Capparuccia L, Prat M, Kerim S, Tamagnone L and Isidoro C: Co-expression of plexin-B1 and Met in human breast and ovary tumours enhances the risk of progression. Cell Oncol 31: 423-436, 2009.

22 Bertotti A and Comoglio PM: Tyrosine kinase signal specificity: lessons from the HGF receptor. Trends Biochem Sci 28: 527533, 2003.

23 Oinuma I, Ishikawa Y, Katoh H and Negishi M: The semaphorin $4 \mathrm{D}$ receptor plexin-B1 Is a GTPase activating protein for R-Ras. Science (80) 305: 862-865, 2004.

24 Oinuma I, Katoh H and Negishi M: Semaphorin 4D/Plexin-B1mediated R-Ras GAP activity inhibits cell migration by regulating beta(1) integrin activity. J Cell Biol 173: 601-613, 2006.

25 Conrotto P, Corso S, Gamberini S, Comoglio PM and Giordano $\mathrm{S}$ : Interplay between scatter factor receptors and $\mathrm{B}$ plexins controls invasive growth. Oncogene 23: 5131-5137, 2004.

26 Wong OG-W, Nitkunan T, Oinuma I, Zhou C, Blanc V, Brown RSD, Bott SRJ, Nariculam J, Box G, Munson P, Constantinou J, Feneley MR, Klocker H, Eccles SA, Negishi M, Freeman A, Masters JR and Williamson M: Plexin-B1 mutations in prostate cancer. Proc Natl Acad Sci USA 104: 19040-19045, 2007.

27 Argast GM, Croy CH, Couts KL, Zhang Z, Litman E, Chan DC and Ahn NG: Plexin B1 is repressed by oncogenic B-Raf signaling and functions as a tumor suppressor in melanoma cells. Oncogene 28: 2697-2709, 2009.

28 Swiercz JM, Worzfeld T and Offermanns S: ErbB-2 andMet reciprocally regulate cellular signaling via plexin-B1. J Biol Chem 283: 1893-1901, 2008. 